\title{
The Chronicle Review
}

http://chronicle.com/free/v55/i30/30b00601.htm

From the issue dated April 3, 2009

\section{Blind Spots}

\section{Humanists must plan their digital future}

By JOHANNA DRUCKER

Stanford University is going through the difficult and highly fraught process of figuring out a plan for the library of the future. Coming on the heels of the "bookless library," touted as the vision for a new engineering library, a preliminary proposal in 2007 to tear down the seismically unsafe Meyer Library and digitize and house off campus most of its 600,000 volumes produced a cry of protest. The utopian ideal, imposed from above, might have met with less resistance had faculty members been involved in the planning from the start. But as charged responses mounted, the administration announced it would delay razing the library. Last fall a faculty committee of the Academic Council proposed its own plan for rethinking the library to the Faculty Senate, which has accepted it with a call for further rethinking by various university groups of how to put the plan into effect.

The new faculty plan is characterized by a key shift in emphasis that is both less charged and more pointed. Rather than envision a "library of the future," it discusses the "future of the library," stressing continuity with an old entity rather than the creation of something brand-new. The distinction exposes what is at stake.

What is at stake here is symptomatic of a national crisis. Libraries have long been characterized as the heart of the research university, the center for scholarship. No one disputes that the aggregated sum of information and material available online, even primary material, has shifted the library's identity. Scholars no longer only visit archives in person; they access library material with immediacy (and increased mediation) online. The need to deal with space crunches and shrinking resources while keeping units critical to a university's mission fully functional is a familiar tale. In California, even at well-endowed Stanford, handling the risks of earthquakes increases the urgency. Buildings have to come down, be replaced, or be repurposed, and libraries are caught wondering how to deal with books. Is this a temporary transition between one traditional library space and a new, updated one? Or do these pressures create an opportunity - or an excuse - to restructure the library? Are all books destined to be condemned to remote storage practically on arrival from their presses, to be read at terminals? What to do?

As the Stanford case illustrates, the vision of the library of the future has been launched first in the sciences, pushed by the conviction that technical information will become more readily and conveniently accessed online - and that the future has arrived. A job advertisement in 2007 for a new head librarian of Stanford's Engineering Library, for example, stated that the new unit would be "a library, but in the most advanced 
definition of that term, and ultimately, as the literatures of engineering disciplines move to digital form, it is envisioned as a bookless facility." Not surprisingly, when the plan to tear down Meyer and replace it with an academic computing service was announced, alarm rose. Too much of the renowned East Asian collection would be available only by sending a page for a book, critics said. Humanists objected that the model of the sciences did not fit their needs. Then came the faculty proposal.

The Stanford faculty recommendations are telling for several reasons, which is why I've bothered to begin my discussion there (or, here, as I enjoy the hospitality of the Stanford Humanities Center as a digital humanities fellow). The faculty committee has made a series of highly reasonable and well-argued proposals. Guiding them is a belief, correct in my opinion and that of most humanists, that books aren't going away, we need them and shall continue to do so for a long time to come, and we cannot pit digital tools against book culture. We must accept the hybrid world of scholarly work and earnestly endeavor to support it.

Among the proposals are also processes for deciding what can or should not be stored remotely and other matters of policy about access and cataloging that would occur whether digital technology was in the mix or not. All envision an expanded service role for new technology that increases efficiency (delivery time for off-site materials) and capabilities (scanned title pages and indices, enhanced cataloging, and enriched metadata). But the sections that stick out include visions of technical solutions to current problems that fall into what I characterize as the "hand-waving magic wand" approach to the future.

Two to-do-list items are particularly striking in the way they point out a critical — and in my opinion terrifying - gap in assigning and accepting responsibility. The faculty report identifies the need for Research Portals and a Browsing Model Project. Both would be ambitious and desirable and would serve scholars of all disciplinary orientations admirably. So where is the problem?

The assumption is that the design and realization of those experimental work environments will occur through the combined efforts of Stanford's applied-technology specialists and its library. The research portals are described as "virtual spaces devoted to research center modules comprised of research portals customized to specific projects, on-line data storage for the materials gathered, and database design for accessing the material in complex ways." The browsing-model project is seen as "a working model in which the experience and intellectual yield of physically browsing books are replicated in an environment comprised of both paper and electronic materials. This will probably involve intense graphics and threedimensional imaging." The faculty committee explained: "We believe that building such a model will convince skeptical faculty about the potential of working with new technologies, and force the library to deliver services that have, up to now, been mainly promises for the future." Even if orchestrating such holographic information fields were readily possible, how should they be designed to support scholarship?

The question cannot be answered in the abstract. The details that will bedevil this and other schemes for the next age of scholarly work and design of the environments to support it are not trivial. And here I come to the crux of my argument. The design of new environments for performing scholarly work cannot be left to the technical staff and to library professionals. The library is a crucial partner in planning and envisioning the future of preserving, using, even creating scholarly resources. So are the technology professionals. But in an analogy with building construction, they are the architects and the contractors. The creation of archives, analytic tools, and statistical analyses of aggregate data in the humanities (and in some other scholarly fields) requires the combined expertise of technical, professional, and scholarly personnel.

The task of modeling an environment for scholarship (not just individual projects, but an environment, with 
a suite of tools for access, use, and research activity) is not a responsibility that can be offloaded onto libraries or technical staffs. I cannot say this strongly or clearly enough: The design of digital tools for scholarship is an intellectual responsibility, not a technical task. After all, what will such "research portals" do? What kinds of work will they be designed to support? Editing? Annotation? Aggregation of leaves of manuscripts scattered at remote institutions? Collaborative writing? Close readings? Data mining? Information display? Multimedia writing? Networked conversation? Publishing? Those are enormous questions, to which no scholar would have the same set of answers as another. No scholar would have the same requirements. But creating boutique, custom solutions on a project-by-project basis is not practical, and the labor involved is too costly. The scope of the task ahead is nothing short of modeling scholarly activity anew in digital media. To answer that challenge, humanists have to do more than wave their hands at the technical professionals.

Scholars in the humanities have been particularly remiss in taking seriously the role they need to play in this project. For years when I was at the University of Virginia, where the library took the lead on digitalhumanities projects, serving as home, sponsor, mentor, and friend to the many research institutes that helped break new ground and establish now-standard practices, faculty members involved in those activities came up repeatedly against a wall of resistance from within their ranks. Humanities, arts, and social-science colleagues repeatedly dismissed digital projects as work for the library community. Most considered the creation of digital materials a technical matter of access, a thing "they" should do and take care of for "us." That attitude contains a grotesque misunderstanding of the basic problem: Unless we scholars are involved in designing the working environments of our digital future, we will find ourselves in a future that doesn't work, without the methods and materials essential to our undertakings. Returning to the architecture analogy, you shouldn't build a new house without dialogue between architect and client. Would you let a contractor determine basic space allocation? Technical experts and library professionals are not mind readers, even in the futuristic film worlds of sci-fi. Design must emerge from the context of use.

Examples of how and why "we" have to play an active role in the design of the scholarly environments of the future abound in the experience of digital humanists - and are more common in the daily experience of scholars trying to perform basic research and writing tasks than many realize. For instance, a number of us who had an opportunity to be part of town meetings reviewing the recommendations for dealing with the shortage of space at the Library of Congress some years ago came up against a basic issue: What version of a work should be digitized as representative of a work? Is Leo Tolstoy's original Russian text of War and Peace sufficient or irrelevant for future generations? Will those generations prefer access to the Louise and Aylmer Maude translation? Or to the more recent translation by Anthony Briggs? Should we digitize the sanitized version of Mark Twain's classics, purged of language now offensive to readers, or the originals that allow the historical distance of culture and vocabulary to register?

In a similar vein, what if another library's only version of Euclid is a copy of Stephen Thomas Hawtrey's An Introduction to the Elements of Euclid used by Bertrand Russell's brother Frank to introduce the future philosopher to its mysteries of mathematics? If the copy is tattered, missing pages, or has other marks showing it was used by a childishly eager Russell, should that bit of history be put aside for the benefits of scanning a clean, new copy of a 10th-grade geometry textbook? In the 2007 article "Inheritance and Loss? A Brief Survey of Google Books," Paul Duguid mordantly observed the shortfalls in Google's plans to digitize library books. He emphasized that the intellectual tasks of vetting editions and assessing scholarly value for generations to come have to be taken into account from the very design of the project, not reverse engineered later.

The technician might suggest that the cleanest, clearest copy that is most legible in OCR (optical character 
recognition) and automated search technologies provides the best return on the digitizing investment. With those criteria, selection is guided by technical requirements and constraints designed into the system. That is not "just" an issue of selection but a fundamental feature of functionality and capability, in other words, the design of the digital environment. The typographical features of the long "s" or radical experiments in graphic layout used by the 20th-century avant-garde - markers of their time and place of production - can be quickly sacrificed in a choice of "legible" (i.e., standardized) fonts. The migration of typeset texts into ASCII streams has been an issue of contention in literary and biographical studies since the advent of the Internet. Such debates underscore the fact that properties of texts are informational, not incidental, to many scholarly projects.

That brings me back to the core of my argument: the problems of designing the environments themselves. What functions do we want a research portal to provide? Three-dimensional imaging and intense graphics are built for scientists and the gaming-entertainment complex. They are radical remediations of traditional forms and formats. All have built-in assumptions about rendering "information" in visual and tractable form. The assumptions of humanists about what can be measured and how are different. They need to bring to bear the same critical interpretation of rhetorical and performative features that are brought to the study of aesthetic texts. Scholars working with the quantitative analysis of cultural materials recognize immediately that the graphical display - granularity, the sequencing of information, or the use of bar charts versus line graphs - of the supposed "information" they are "mining" represent rhetorical variables.

The metaphors of mining are as mechanistic as old concepts of the New Critics about "getting" meaning from a work - as if it could be extracted in buckets and removed, usually by students relieved to be able to leave the nasty, troubling body of the text behind. But of course, we want to be troubled: It is precisely the capacity for "troubling" that makes a text engaging, provocative, productive. Knowledge does not exist outside of circumstances of use or independent of its material means of expression. The history of literary and cultural studies in the last 50 years is a sequence of critical interventions in the assumption that a text is a static thing.

Many humanities principles developed in hard-fought critical battles of the last decades are absent in the design of digital contexts. Here is a short list: the subjectivity of interpretation, theoretical conceptions of texts as events (not things), cross-cultural perspectives that reveal the ideological workings of power, recognition of the fundamentally social nature of knowledge production, an intersubjective, mediated model of knowledge as something constituted, not just transmitted. For too long, the digital humanities, the advanced research arm of humanistic scholarly dialogue with computational methods, has taken its rules and cues from digital exigencies.

If we are interested in creating in our work with digital technologies the subjective, inflected, and annotated processes central to humanistic inquiry, we must be committed to designing the digital systems and tools for our future work. Nothing less than the way we understand knowledge and our tasks as scholars are at stake. Software and hardware only put into effect the models structured into their design.

Moreover, university administrators need to see such work as more valuable than they have to date. Faculty members and graduate students committed to remodeling knowledge with innovative approaches to scholarship have to be supported. With rare exceptions, the work, too easily seen as tool-building, has occurred at the edges of digital projects and is usually financed with grants. That does not result in approaches that can be generalized beyond specific projects.

Unless scholars in the humanities help design and model the environments in which they will work, they 
will not be able to use them. Tools developed for PlayStation and PowerPoint, Word, and Excel will be as appropriate to our intellectual labors as a Playskool workbench is to the chores of a real plumber. I once bought a very beautiful portable Olivetti typewriter because an artist friend of mine said it was so elegantly designed that it had been immediately put into the Museum of Modern Art collection. The problem? It wasn't designed for typing. Any keyboardist with any skill at all constantly clogged its keys. A thing of beauty, it was a pain forever. I finally threw it from the fourth-floor tower of Wurster Hall at the University of California at Berkeley. Try doing that with the interface to your university library. Now reflect on who is responsible for getting it to work as an environment that supports scholarship.

We face a critical juncture. Leaving it to "them" is unfair, wrongheaded, and irresponsible. Them is us.

Johanna Drucker is a professor of information studies at the University of California at Los Angeles. This year she is a Digital Humanities Fellow at the Stanford Humanities Center.

http://chronicle.com

Section: The Chronicle Review

Volume 55, Issue 30, Page B6

Copyright (c) 2009 by The Chronicle of Higher Education

$\underline{\text { Subscribe I }}$ About The Chronicle I Contact us I Terms of use I Privacy policy I Help 